\title{
CHROMOSOME NUMBERS OF SCHISTIDIUM (GRIMMIACEAE, BRYOPHYTA)
}

\author{
ХРОМОСОМНЫЕ ЧИСЛА ВИДОВ РОДА SCHISTIDIUM \\ (GRIMMIACEAE, BRYOPHYTA) \\ I.S. DANILKIV ${ }^{1}$, E.A. IGNATOVA ${ }^{2} \&$ O.V. LOBACHEVSKA ${ }^{1}$ \\ И.С. ДАНИЛКИВ ${ }^{1}$, Е.А. ИГНАТОВА ${ }^{2}$, О.В. ЛОБАчЕВСКАЯ ${ }^{1}$
}

Abstract

Chromosome numbers were reported for the genus Schistidium mostly before it was critically revised by H.H. Blom, who strongly changes species-level taxonomy of this genus. Old data on chromosome counts in Schistidium thus became difficult to use. In the present study 106 specimens with chromosome counts were revised. Among them we identified 13 species (S. apocarpum, S. crassipilum, S. dupretii, $S$. elegantulum, S. flaccidum, S. helveticum, S. lancifolium, S. papillosum, S. platyphyllum, S. pulchrum, S. robustum, S. trichodon var. nutans, S. submuticum), thus providing for many 'narrow' species the chromosome counts (all based on meiosis) for the first time. Most of them have $n=13$, while $n=26$ seems to be a characteristic of most specimens of $S$. apocarpum s. str. and one of S. submuticum, but other specimens of both these species have $n=13$ as well.

Резюме

Хромосомные числа исследовались у видов рода Schistidium преимущественно до его ревизии Г.Бломом, в результате которой объем и понимание видов значительно изменились. Старые данные по хромосомным числам рода стало практически невозможно использовать. В связи с этим были переизучены 106 образцов с определенными хромосомными числами, среди которых выявлены 13 видов (S. apocarpum, S. crassipilum, S. dupretii, S. elegantulum, S. flaccidum, S. helveticum, S. lancifolium, S. papillosum, S. platyphyllum, S. pulchrum, S. robustum, S. trichodon var. nutans, S. submuticum), для многих из которых хромосомные числа (основанные на изучении мейоза) приводятся впервые. Большинство видов имеет $\mathrm{n}=13$, тогда как $\mathrm{n}=26$ выявлен только у $S$. apocarpum s. str. (большинство образцов) и $S$. submuticum (один образец). Для обоих этих видов, однако имеются и расы с n=13.

KEYWORDS: Schistidium, Grimmiaceae, Bryophyta, chromosome numbers

The taxonomy of the genus Schistidium provides the case of the major incongruence of approaches among the recent authors. Bremer (1980a, b; 1981) published a world monograph, where she accepted 12 species in the genus, among them 6 species in Europe and 4 of them from S. apocarpum complex. Blom (1996) recognized 31 species in the $S$. apocarpum complex in Norway and Sweden. The primary negative reaction on this catastrofic over-splitting shifted soon afterward to a successful application of Blom concept in regional floristic studies (cf. Ochyra et al., 2003; Smith, 2004). Recent checklist of the Europe (Hill et al., 2005), the North Europe and North Asia (Ignatov, Afonina, Ignatova et al., 2006), as well the treatment of the genus in

1 - Institute of Ecology of the Carpathians, National Academy of Sciences of Ukraine, Dept. of Plant Ecomorphogenesis, Stefanyka Str., 11, Lviv 79000, Ukraine; e-mail: morphogenesis@mail.lviv.ua

2 - Moscow State University, Biological Faculty, Geobotany Dept., Moscow 119991 Russia; e-mail: arctoa@list.ru 
Table 1. Chromosome numbers of Schistidium species (specimens in LWKS)

\begin{tabular}{|c|c|c|c|c|c|}
\hline Species & Locality & $\mathrm{n}=$ & Coll. date & Collector & № \\
\hline apocarpum & Belarus, Grodno Prov., Grodno & 13 & 11.X.1972 & O.I.Vysotska & 18 \\
\hline apocarpum & Belarus, Grodno Prov., Zarubichi & 13 & 10.X.1972 & O.I.Vysotska & 31 \\
\hline apocarpum & Belarus, Grodno Prov., Zarubichi & 13 & 10.X.1972 & O.I.Vysotska & 33 \\
\hline apocarpum & Belarus, Grodno Prov., Zarubichi & 13 & 10.X.1972 & O.I.Vysotska & 35 \\
\hline apocarpum & Belarus, Grodno Prov., Zarubichi & 26 & 10.X.1972 & O.I.Vysotska & 65 \\
\hline apocarpum & Belarus, Minsk Prov., Myadel & 13 & 25.IX.1973 & O.I.Vysotska & 19 \\
\hline apocarpum & Belarus, Minsk Prov., Myadel & 26 & 23.IX.1973 & O.I.Vysotska & 63 \\
\hline apocarpum & Belarus, Minsk Prov., Myadel Distr., Brusi & 26 & 25.IX.1973 & O.I.Vysotska & 80 \\
\hline apocarpum & Belarus, Minsk Prov., Myadel Distr., Pasinki & 26 & 22.IX.1973 & O.I.Vysotska & 77 \\
\hline apocarpum & Belarus, Minsk Prov., Myadel Distr., Pasinki & 13 & 23.IX.1973 & O.I.Vysotska & 83 \\
\hline apocarpum & Latvia, Pabazhi & 26 & 11.IX.1967 & L.M.Fetisova & 50 \\
\hline apocarpum & Latvia, Sigulda & 26 & 15.IX.1968 & L.M.Fetisova & 6 \\
\hline apocarpum & Latvia, Smiltine & 26 & 18.IX.1975 & I.S.Danilkiv & 68 \\
\hline apocarpum & Latvia, Smiltine & 26 & 18.IX.1975 & I.S.Danilkiv & 79 \\
\hline apocarpum & Latvia, Smiltine & 26 & 18.IX.1975 & I.S.Danilkiv & 96 \\
\hline apocarpum & Latvia, Smiltine & 26 & 18.IX.1972 & I.S.Danilkiv & 97 \\
\hline apocarpum & Lietuva, Alitus Distr., Daugay & 26 & 01.X.1973 & I.S.Danilkiv & 74 \\
\hline apocarpum & Lietuva, Shaulyay & 26 & 12.IX.1971 & I.S.Danilkiv & 3 \\
\hline apocarpum & Lietuva, Shaulyay & 26 & 23.IX.1966 & I.S.Danilkiv & 61 \\
\hline apocarpum & Lietuva, Shaulyay & 26 & 12.IX.1971 & I.S.Danilkiv & 66 \\
\hline apocarpum & Lietuva, Vilnyus & 26 & 12.X.1971 & I.S.Danilkiv & 5 \\
\hline apocarpum & Lietuva, Vilnyus & 26 & 12.XI.1971 & I.S.Danilkiv & 60 \\
\hline apocarpum & Lietuva, Vilnyus & 26 & 09.X.1973 & I.S.Danilkiv & 70 \\
\hline apocarpum & Lietuva, Vilnyus & 26 & 09.X.1973 & I.S.Danilkiv & 71 \\
\hline apocarpum & Lietuva, Vilnyus & 26 & 09.X.1973 & I.S.Danilkiv & 72 \\
\hline apocarpum & Lietuva, Vilnyus & 26 & 09.X.1973 & I.S.Danilkiv & 75 \\
\hline apocarpum & Lietuva, Vilnyus & 26 & 09.X.1973 & I.S.Danilkiv & 76 \\
\hline apocarpum & Lietuva, Vilnyus & 26 & 16.X.1971 & I.S.Danilkiv & 94 \\
\hline apocarpum & Russia, Kaliningrad Prov. & 26 & 1.IX.1974 & I.S.Danilkiv & 4 \\
\hline apocarpum & Russia, Karelia, Sortavala & 26 & 10.IX.1986 & E.M.Lesnyak & 53 \\
\hline apocarpum & Russia, Karelia, Sortavala & 26 & 10.IX.1986 & E.M.Lesnyak & 55 \\
\hline apocarpum & Ukraine, Ivano-Frankivsk & 13 & 12.X.1968 & E.M.Lesnyak & 89 \\
\hline apocarpum & Ukraine, Ivano-Frankivsk Prov., Vorokhta & 13 & 24.IX.1970 & O.I.Vysotska & 20 \\
\hline apocarpum & Ukraine, Ivano-Frankivsk Prov., Yaremche & 26 & 15.X.1983 & I.S.Danilkiv & 58 \\
\hline apocarpum & Ukraine, Lviv & 26 & 01.XI.1983 & I.S.Danilkiv & 56 \\
\hline apocarpum & Ukraine, Lviv & 26 & 01.XI.1983 & I.S.Danilkiv & 57 \\
\hline apocarpum & Ukraine, Lviv & 26 & 04.IX.1969 & A.Skalska & 62 \\
\hline apocarpum & Ukraine, Lviv & 13 & 13.IV.1980 & E.M.Lesnyak & 90 \\
\hline apocarpum & Ukraine, Lviv & 13 & 13.IV.1980 & E.M.Lesnyak & 91 \\
\hline apocarpum & Ukraine, Lviv & 26 & 13.IV.1980 & E.M.Lesnyak & $91 \mathrm{a}$ \\
\hline apocarpum & Ukraine, Lviv Prov., Borislav & 13 & 01.XI.1969 & A.Skalska & 14 \\
\hline apocarpum & Ukraine, Lviv Prov., Borislav & 26 & 15.X.1969 & E.M.Lesnyak & 48 \\
\hline apocarpum & Ukraine, Lviv Prov., Borislav & 26 & 15.X.1969 & E.M.Lesnyak & 59 \\
\hline apocarpum & Ukraine, Lviv Prov., Borislav & 26 & 15.X.1969 & E.M.Lesnyak & 67 \\
\hline apocarpum & Ukraine, Lviv Prov., Borislav & 26 & 01.XI.1969 & A.Skalska & 7 \\
\hline apocarpum & Ukraine, Lviv Prov., Skole Distr., Dubyna/Kamyanka & 26 & 22.IX.1968 & O.I.Vysotska & 51 \\
\hline apocarpum & Ukraine, Lviv Prov., Skole Distr., Kamyanka & 26 & 30.IX.1969 & O.I.Vysotska & 69 \\
\hline apocarpum & Ukraine, Lviv Prov., Yaniv to Stradch & 26 & 15.X.1969 & E.M.Lesnyak & 73 \\
\hline apocarpum* & Georgia, Bakuriani & 26 & 22.IX.1967 & U.K.Mamatkuloy & v95 \\
\hline apocarpum* & Georgia, Batumi & 13 & 10.X.1972 & L.M.Fetisova & 39 \\
\hline apocarpum $*$ & Georgia, Batumi & 13 & 23.XI.1965 & L.M.Fetisova & 43 \\
\hline apocarpum* & Latvia, Lelupe & 13 & 12.IX.1968 & L.M.Fetisova & 15 \\
\hline apocarpum* & Latvia, Smiltine & 26 & 18.IX.1975 & I.S.Danilkiv & 78 \\
\hline apocarpum* & Ukraine, Ivano-Frankivsk Prov., Kosiv & 13 & 18.X.1969 & E.M.Lesnyak & 13 \\
\hline
\end{tabular}


apocarpum* Ukraine, Lviv

apocarpum* Ukraine, Lviv

apocarpum* Ukraine, Lviv Prov., Borislav

apocarpum* Ukraine, Lviv Prov., Borislav

apocarpum* Ukraine, Lviv Prov., Skole Distr., Dubyna

Ukraine, Lviv Prov., Skole Distr., Dubyna/Kamyanka 13

apocarpum*

apocarpum*

apocarpum*

apocarpum*

apocarpum*

crassipilum

crassipilum

crassipilum

crassipilum*

crassipilum* Russia, Adygeya, Dagestanskaya

crassipilum* Russia, Adygeya, Dakhovskaya

crassipilum* Russia, Adygeya, Rufabgo Creek

crassipilum* Russia, Adygeya, Rufabgo Creek

crassipilum* Russia, Adygeya, Rufabgo Creek

crassipilum* Ukraine, Lviv Prov., Borislav

dupretii Ukraine, Ivano-Frankivsk

dupretii

dupretii

elegantulum

elegantulum

elegantulum

elegantulum

elegantulum

elegantulum

flaccidum

flaccidum*

helveticum

helveticum

lancifolium

lancifolium

lancifolium

lancifolium

lancifolium

lancifolium

lancifolium*

trichodon var.

nutans Ukraine, Ivano-Frankivsk Prov., Chornogora Range

papillosum Ukraine, Lviv Prov., Skole Distr., Kamyanka

platyphyllum Russia, Altay Republic, Ust-Sema

platyphyllum*Russia, Altay Republic, Ust-Sema

pulchrum Ukraine, Chernivtsi Prov., Shepit

robustum Ukraine, Lviv Prov., Borislav

robustum* Ukraine, Ternopil Prov., Ostapye

robustum ${ }^{1}$ Ukraine, Lviv Prov., Borislav

submuticum Estonia, Akhij River

submuticum Estonia, Tallinn

submuticum Estonia, Tallinn

submuticum

submuticum
Latvia, Plyavinyas

Russia, Lipetsk Prov., Galichya Gora Reserve
13

13

26

13

13

13

.

3

1.XI.1983 O.V.Lobachevska64 29.VIII.1969 E.Sytnik 8

15.X.1969 E.M.Lesnyak 47

01.XI.1969 A.Skalska 49

28.IX.1971 O.I.Vysotska 28

22.IX.1968 O.I.Vysotska 34

22.VIII.1968 O.I.Vysotska 40

22.IX.1968 O.I.Vysotska 41

30.IX.1969 O.I.Vysotska 27

10.IX.1965 A.S.Lazarenko 2

06.XI.1974 I.S.Danilkiv 101

20.XI.1974 I.S.Danilkiv 85

20.XI.1974 I.S.Danilkiv 98

29.X.1955 L.M.Fetisova 24

26.XI.1974 I.S.Danilkiv 92

18.XI.1974 I.S.Danilkiv 93

25.XI.1974 I.S.Danilkiv 102

06.XI.1974 I.S.Danilkiv 104

20.XI.1974 I.S.Danilkiv 84

15.X.1969 E.M.Lesnyak 45

25.VIII.1967 E.M.Lesnyak 32

21.X.1970 O.I.Vysotska 29

01.XI.1969 A.Skalska 12

26.XI.1974 I.S.Danilkiv 86

18.XI.1974 I.S.Danilkiv 100

18.XI.1974 I.S.Danilkiv 16

18.XI.1974 I.S.Danilkiv 21

18.XI.1974 I.S.Danilkiv 22

20.XI.1974 I.S.Danilkiv 99

30.III.1965 U.K.Mamatkulov1

22.X.1981 O.I.Vysotska 30

12.VII.1977 O.I.Vysotska 103

12.VII.1977 O.I.Vysotska 105

15.X.1969 O.I.Vysotska 81

22.IX.1967 U.K.Mamatkulov82

27.IX.1966 L.M.Fetisova 88

25.IX.1970 O.I.Vysotska 106

15.X.1983 I.S.Danilkiv 42

30.IX.1966 O.I.Vysotska 87

12.IX.1964 A.S.Lazarenko 10

05.X.1966 A.S.Lazarenko 46

22.IX.1968 E.M.Lesnyak 23

14.VIII.1977 O.I.Vysotska 37

14.VIII.1977 O.I.Vysotska 36

23.IX.1966 A.S.Lazarenko 44

27.X.1964 E.M.Lesnyak 17

24.VI.1975 I.S.Danilkiv 11

27.IX.1964 E.M.Lesnyak 9

29.V.1967 L.M.Fetisova 38

24.IX.1968 L.M.Fetisova 26

24.IX.1968 L.M.Fetisova 52

27.IX.1966 L.M.Fetisova 25

28.IX.1992 Vyshegorodskikh 54

\footnotetext{
${ }^{1}$ - this specimens has $S$. apocarpum as admixture, but premature capsules that likely were used for chromosome count belong to plants of $S$. robustum.
} 
new bryophyte flora of North America (McIntosh, 2007) followed Blom's narrow concept for this genus, accepting 42, 42, and 30 species correspondingly. Finally, Goryunov et al. (2007) supported Blom's approach by molecular phylogenetic data, and subsequent studies with the broader set of data just confirm this point.

This changes in taxonomy, of course, urge the complete revision of the genus: old data of distribution of species can not be used any more (at least in most cases). Similarly, old data of chromosome numbers need a complete revision. By this reason we undertook this revision of collection of 106 specimens with identified numbers in LWKS. Part of them was published previously by Lazarenko et al. (1971).

Results of the revision of the collections is given in Table 1. All identificatios were made based of meiosis study, the details of method explained in Lazarenko et al. (1971).

A certain disadvantage of this method in our case is that the plants were collected with premature capsules, which in some cases makes the species identification not very certain, as peristome and mature spores were unavailable for study. Such specimens are marked in the table with asterisk $(*)$, indicating somewhat less reliable determinations. In many specimens, however, few more mature capsules occur in collections, allowing identification as certain as can be done for optimally collected material.

Few specimens represented by scanty material with only very young capsules are not included in the present list.

Most of specimens have $n=13$, while $n=26$, identified for as many as for 45 specimens, and
44 of them belong to $S$. apocarpum, while only one to $S$. submuticum. Both latter species, however, have $n=13$ as well, in 20 and 4 specimens correspondingly.

\section{ACKNOWLEDGEMENTS}

The study was partly supported by RFBR 07 04-00013.

\section{LITERATURE CITED}

BLOM, H.H. 1996. A revision of the Schistidium apocarpum complex in Norway and Sweden. - Bryoph. Bibl. 49: 1-333.

BREMER, B. 1980a. A taxonomic revision of Schistidium (Grimmiaceae, Bryophyta) 1. - Lindbergia 6: 1-16.

BREMER, B. 1980b. A taxonomic revision of Schistidium (Grimmiaceae, Bryophyta) 2. - Lindbergia 6: 89-117.

BREMER, B. 1981. A taxonomic revision of Schistidium (Grimmiaceae, Bryophyta) 3. - Lindbergia 7: 73-90.

GORYUNOV, D.V., E.A. IGNATOVA, M.S. IGNATOV, I.A.MILYUTINA \& A.V. TROITSKY 2007. Support from DNA data for a narrow species concept in Schistidium (Grimmiaceae, Musci). - J. Bryol. 29: 98-103.

HILL, M.O., N. BELL, M.A. BRUGGEMAN-NANNENGA, M. BRUGUÉS, M.J. CANO, J. ENROTH, K.I. FLATBERG, J.-P. FRAHM, M.T. GALLEGO, R. GARILLETI, J. GUERRA, L. HEDENAS, D.T. HOLYOAK, J. HYVÖNEN, M.S. IGNATOV, F. LARA, V. MAZIMPAKA, J. MUÑOZ \& L. SÖDERSTRÖM 2006. An annotated checklist of the mosses of Europe and Macaronesia. - J. Bryol. 28: 198-267.

IGNATOV M.S., O.M. AFONINA, E.A. IGNATOVA et al. 2006. Check-list of mosses of East Europe and North Asia. Arctoa 15: 1-130.

[LAZARENKO, A.S., E.I. VYSOTSKAYA \& E.N. LESNYAK] ЛАЗАРЕНКО, А.С., Е.И. ВЫСОЦКАЯ, Е.Н. ЛЕСНЯК 1971. Атлас хромосом лиственных мхов CCCP. - [Atlas of chromosomes of mosses of the USSR] Киев, Наукова думка [Kiev, Naukova dumka], 144 pp.

OCHYRA, R, J. ŻARNOWIEC \& H. BEDNAREK-OCHYRA 2003. Census catalogue of Polish mosses. - Kraków: Institute of Botany, Polish Academy of Sciences. 372 pp.

SMITH, A.J.E. 2004. The moss flora of Britain and Ireland. 2 ed. - Cambridge: Cambridge University Press. 1012 pp. 